\title{
Editorial commentary: Another case report on congenital mesoblastic nephroma - why?
}

In the past 25 years, congential mesoblastic nephroma (CMN) has emerged as a fascinating lesion. First described by Bolande in 1967 as a renal tumor, it was at that time called a hamartomatous renal lesion by Wigger at the Babies Hospital. All pediatric radiologists rapidly learned that $\mathrm{CMN}$ was the renal tumor of the newborn - that Wilms' tumor did not exist in newborn.
Further, CMN needed only surgery and metastases did not occur.

Every one of these statements has proved to be incorrect, because:

1. CMN exists beyond the newborn, even into adult life.

2. True Wilms' tumor does occur in infants, even newborns.

3. CMN has exhibited spread to lung, bone, heart, and brain.
It is important to emphasize that $\mathrm{CMN}$ cannot be differentiated from Wilms' tumor by any imaging modality to date. It also is the most common renal tumor in children to present with hypercalcemia [1].

W. E. Berdon

\section{Congenital mesoblastic nephroma metastatic to the brain: a report of two cases}

\author{
A. E. Schlesinger ${ }^{1}$, N.S. Rosenfield ${ }^{2}$, V.P. Castle ${ }^{3}$, R. Jasty ${ }^{3}$ \\ ${ }^{1}$ Department of Diagnostic Imaging, Texas Children's Hospital, Houston, TX, USA \\ ${ }^{2}$ Department of Radiology, Memorial Sloan-Kettering Cancer Center, New York, NY, USA \\ ${ }^{3}$ Department of Pediatrics, University of Michigan Hospitals, Ann Arbor, MI, USA
}

Received: 2 February 1995/Accepted: 4 April 1995

\begin{abstract}
Congenital mesoblastic nephroma was originally believed to be a universally benign neoplasm. More recently, aggressive congenital mesoblastic nephromas have been described with local recurrence and/or metastases. We report two patients with documented congenital mesoblastic nephroma which later metastasized to the brain.
\end{abstract}

Congenital mesoblastic nephroma (CMN) was first described by Bolande et al. [1] as a benign lesion. Subsequent studies have described more aggressive behavior with local recurrence or metastatic disease [2-8]. This has led to a reappraisal of $\mathrm{CMN}$ and to recognition of a more aggressive, atypical form of this disease [9]. We describe two cases of CMN with metastases to the brain; these patients have been previously reported in the pathology literature $[10,11]$. Brain metastases from $\mathrm{CMN}$ and their imaging findings have not been previously described in the radiology literature. These cases

Correspondence to: A.E. Schlesinger, 1323 Brynnwood Dr., St. Louis, MO 63124, USA are reported to bring to radiologist's attention the possibility of brain metastases from CMN.

\section{Case reports}

Case 1

A male infant was born at term to a G1P0 mother whose pregnancy was complicated by a known seizure disorder. A routine prenatal ultrasound examination revealed a left renal mass. This was confirmed after birth by a computed tomography (CT) scan, which demonstrated a large, heterogeneous intrarenal mass (Fig.1). A left nephrectomy was performed on the tenth postnatal day, and pathologic examination was consistent with CMN with a mixed classical and cellular pattern. At 7 months of age, the patient presented with irritability, vomiting, decreased appetite, and decreased movement of the right extremities. Physical examination revealed a head circumference at the 95 th percentile with a bulging anterior fontanel and paresis of the right extremities. Magnetic resonance (MR) imaging demonstrated a large left cerebral mass with midline shift and enlargement of the contralateral lateral ventricle (Fig. 2). These was no imaging evidence of recurrence of the primary tumor. A craniotomy was performed with gross total resection of the tumor. Pathologic examination was consistent with metastatic $\mathrm{CMN}$ with the same histological findings as 